\title{
Identification of key genes for laryngeal squamous cell carcinoma using weighted co-expression network analysis
}

\author{
XIAO-TIAN LI

\begin{abstract}
Department of Otolaryngology-Head and Neck Surgery, The First Hospital of China Medical University,
\end{abstract} \\ Shenyang, Liaoning 110001, P.R. China
}

Received February 10, 2015; Accepted February 19, 2016

DOI: $10.3892 / 01.2016 .4378$

\begin{abstract}
Laryngeal squamous cell carcinoma (LSCC) is the most common malignant tumor in the head and neck, and can seriously affect the daily life of patients. To study the mechanisms of LSCC, the microarray of GSE51958 was analyzed in the present study. GSE51958 was downloaded from Gene Expression Omnibus, and included a collection of LSCC tissue samples and matched adjacent non-cancerous tissue samples from 10 patients. Differentially-expressed genes (DEGs) were identified using limma package. Next, a weighted co-expression network was constructed for the DEGs by WGCNA package in R. Modules of the weighted co-expression network were obtained through constructing a hierarchical clustering tree using the hybrid dynamic shear tree method. Using the clusterProfiler package, the potential functions of DEGs in the modules correlated with LSCC were predicted by pathway enrichment analysis. In total, 959 DEGs were screened from the LSCC samples compared with the adjacent non-cancerous samples, including 553 upregulated and 406 downregulated genes. The appointed black, brown, gray, pink and yellow modules were screened for the DEGs in the weighted co-expression network. For the DEGs in the brown and yellow modules, the enriched pathways were cytokine-cytokine receptor interaction and metabolic pathways, respectively. The DEGs in the pink module were involved in the majority of pathways. With high connectivity degrees in the pink module, TPX2, microtubule-associated (TPX2; degree, 25), minichromosome maintenance complex component 2 (MCM2; degree, 25), ubiquitin-like with PHD and ring finger domains 1 (UHRF1; degree, 22), cyclin-dependent kinase 2 (CDK2; degree, 20) and protein regulator of cytokinesis 1 (PRCl; degree, 20)
\end{abstract}

Correspondence to: Dr Xiao-Tian Li, Department of Otolaryngology-Head and Neck Surgery, The First Hospital of China Medical University, 155 Nanjing Bei Street, Shenyang, Liaoning 110001, P.R. China

E-mail: xiaotianlixtl@163.com

Key words: laryngeal squamous cell carcinoma, module analysis, weighted co-expression network, differentially-expressed genes, pathway enrichment analysis may be involved in LSCC. Overall, In conclusion, from the integrated bioinformatics analysis of genes that may be associated with LSCC, 959 DEGs were obtained from LSCC samples compared with adjacent non-cancerous samples, and TPX2, MCM2, UHRF1, CDK2 and PRC1 were found to hold a possible association with the disease.

\section{Introduction}

Laryngeal squamous cell carcinoma (LSCC) is the most prevalent malignant tumor in the head and neck $(1,2)$. Due to the key functions of the larynx in respiration and phonation, LSCC can seriously affect the daily life of patients (3). At present, surgical intervention, chemotherapy and radiotherapy can be used in the treatment of primary LSCC, however, these methods have poor effects in advanced patients (4). Thus, there is an urgent requirement to identify genes involved in LSCC and to develop novel therapeutic schedules.

Cox proportional hazards analysis has shown that cyclin-dependent kinase inhibitor $2 \mathrm{~A}$ point mutation is associated with disease relapse and mortality, thus, it may serve as a key biomolecular indicator in $\operatorname{LSCC}(5,6)$. Downregulated human leukocyte antigen class I can reduce the survival time of patients with LSCC and can be used as an independent prognostic marker (7). The overexpression and/or co-overexpression of cyclin D1 and cyclin-dependent kinase 4 (CDK4) may be implicated in the biological behavior of LSCC and have a valuable prognostic significance $(8,9)$. The expression of S100 calcium binging protein A2 is associated with cytokeratin expression, cell commitment to squamous differentiation and overall survival in LSCC (10). Recombinant lentivirus mediated siRNA silencing of matrix metallopeptidase $2(M M P-2)$ can suppress growth and invasion of LSCC, therefore, $M M P-2$ may function in the gene therapy of $\operatorname{LSCC}(11,12)$. Overexpressed stomatin-like protein 2 promotes cell growth, tumorigenicity and adhesion, and has a correlation with clinical stage in human LSCC $(13,14)$. In spite of studies performed to investigate LSCC, the mechanisms of LSCC remain unclear.

In the present study, to further reveal the mechanisms of LSCC, differentially-expressed genes (DEGs) were screened. Additionally, a weighted co-expression network was constructed for the DEGs and a module analysis was conducted. Additionally, the potential functions of DEGs in modules were analyzed by pathway enrichment analysis. 

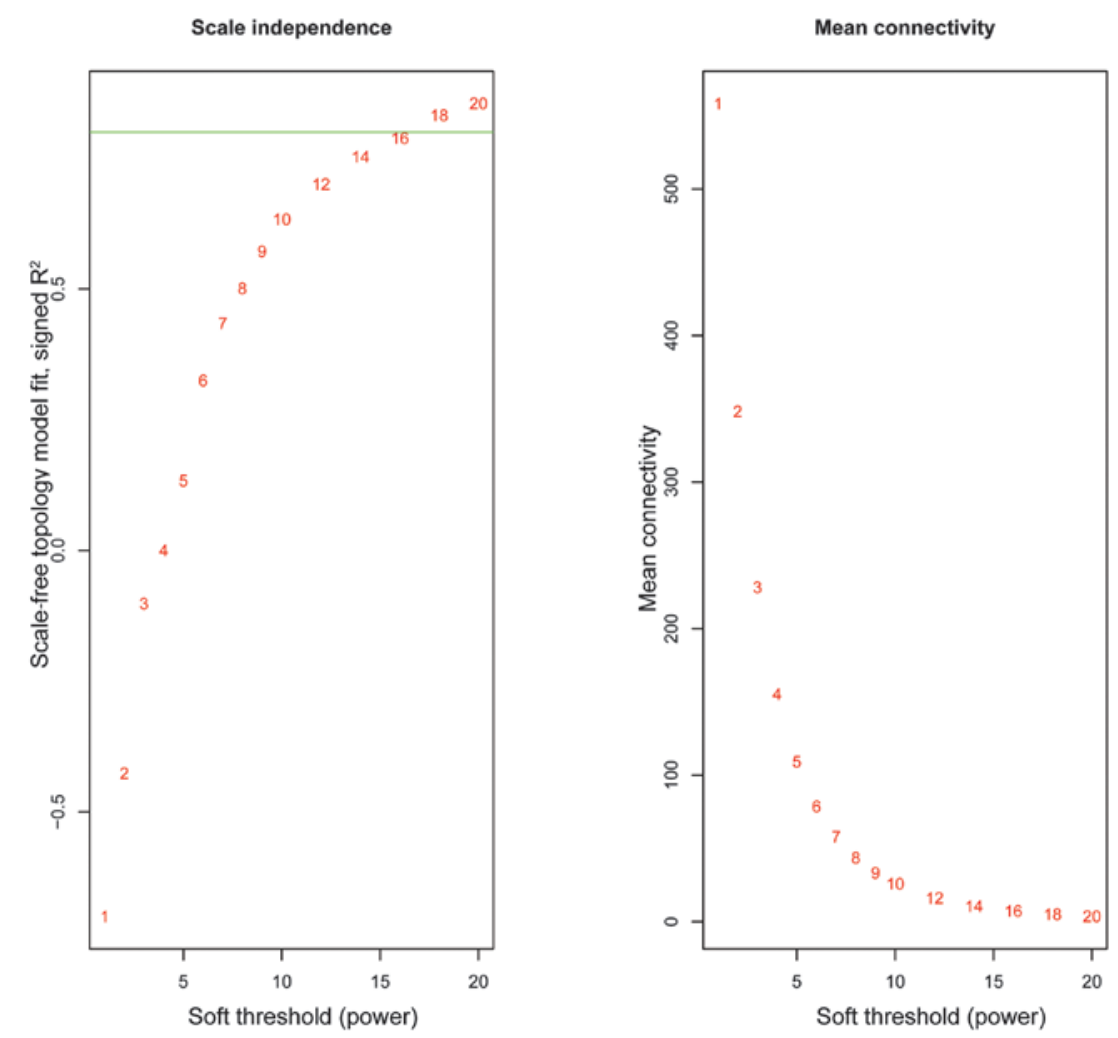

Figure 1. Selection of the weighting coefficient.

\section{Materials and methods}

Microarray data. The expression profile of GSE51958, which was downloaded from Gene Expression Omnibus (http://www.ncbi.nlm.nih.gov/geo/), was based on the platforms of GPL17869 CytoSure Human Custom Oligonucleotide 4x180 k Array v.031035 and GPL17870 CytoSure Human Custom Oligonucleotide 4x180 k Array v.025990. GSE51958 included a collection of LSCC tissue samples and matched adjacent non-cancerous tissue samples from 10 patients.

DEG screening. Once GSE51958 was downloaded, normalized microarray data was obtained. The probes with low expression in $\geq 20$ microarrays were excluded. According to the annotation files, probes that were corresponding to any genes were eliminated. Subsequently, the limma (linear models for microarray data) package (15) (http://www.bioconductor.org) was used to screen the DEGs between the LSCC samples and matched adjacent non-cancerous samples. The adjusted P-value of $<0.05$ and llogfold change $(\mathrm{FC}) \mid \geq 1$ were used as the cut-off criteria.

Weighted co-expression network construction. The WGCNA package (16) in R was used to construct weighted co-expression networks for the DEGs. Briefly, Pearson's correlation coefficients between the DEGs were calculated using their expression matrices. The correlation coefficient of $\geq 0.8$ was defined as the weighting coefficient.

A hierarchical clustering tree was constructed for the DEGs using the hybrid dynamic shear tree method (17), and branches of the clustering tree represented the gene modules. Each module had to be involved with at least 10 genes. Afterwards, the feature vector of each module (module eigengenes) was calculated and cluster analysis was performed for the modules. The closed modules (difference of feature vectors $<0.15$ ) were merged into new modules. Furthermore, correlation analysis between modules and LSCC was performed. Gene significance (GS) and module significance (MS; the mean value of all GS values) were calculated. The module with the highest MS had a closer correlation with LSCC.

Pathway enrichment analysis. The Kyoto Encyclopedia of Genes and Genomes (KEGG) is a reference knowledge base involving systems information, genomic information and chemical information (18). Using the clusterProfiler package (19) (http://bioconductor.org/packages/release/bioc/html/clusterProfiler.html), KEGG pathway enrichment analyses were conducted separately for the DEGs in the modules. A P-value of $<0.1$ was used as the cut-off criterion.

\section{Results}

$D E G$ analysis. When compared with the adjacent non-cancerous samples, a total of 959 DEGs were screened from the LSCC samples, including 553 upregulated and 406 downregulated genes. Evidently, there were more upregulated genes than downregulated genes.

Weighted co-expression network construction. The weighted co-expression network was constructed and the weighting coefficient was set as 17 (Fig. 1). Modules were identified from the weighted co-expression network. After these closed 
Table I. Statistics for the five modules (black, brown, gray, pink and yellow modules).

\begin{tabular}{|c|c|c|c|c|c|}
\hline \multirow[b]{2}{*}{ Module } & \multicolumn{2}{|c|}{ Size } & \multicolumn{2}{|c|}{ ME-LSCC correlation } & \multirow[b]{2}{*}{ MS } \\
\hline & Upregulated & Downregulated & Absoulute coefficient & P-value & \\
\hline Black & 40 & 1 & 0.81 & $1.82 \times 10^{-5}$ & 0.68 \\
\hline Pink & 414 & 104 & 0.86 & $1.21 \times 10^{-6}$ & 0.69 \\
\hline Brown & 55 & 203 & -0.85 & $2.36 \times 10^{-6}$ & 0.69 \\
\hline Yellow & 36 & 90 & -0.84 & $4.26 \times 10^{-6}$ & 0.69 \\
\hline Gray & 8 & 8 & -0.92 & $9.84 \times 10^{-9}$ & 0.65 \\
\hline
\end{tabular}

ME, module eigengenes; MS, module significance; LSCC, laryngeal squamous cell carcinoma; ME-LSCC correlation, pearson correlation coefficients between ME and LSCC.

Table II. Pathways enriched for differentially-expressed genes in the pink, brown and yellow module.

\begin{tabular}{|c|c|c|c|c|c|c|}
\hline Module & Category & Term & Description & Gene number & Gene & P-value \\
\hline \multirow[t]{8}{*}{ Pink } & KEGG & 03030 & DNA replication & 12 & $\begin{array}{l}\text { FEN1, MCM2, MCM3, MCM4, } \\
\text { RFC5, RFC4, DNA2, POLA2, } \\
\text { RNASEH2A, PRIM2, POLE2, } \\
\text { PRIM1 }\end{array}$ & $6.02 \times 10^{-10}$ \\
\hline & KEGG & 04110 & Cell cycle & 19 & $\begin{array}{l}\text { CDK4, CDK2, MCM2, PRKDC, } \\
\text { MCM3, MCM4, CDC25B, ORC1, } \\
\text { PKMYT1, CDC25A, SKP2, CDC20, } \\
\text { TTK, MAD2L1, CDC45, CHEK1, } \\
\text { CCNB1, CCNE1, CDK6 }\end{array}$ & $2.05 \times 10^{-8}$ \\
\hline & KEGG & 05222 & Small cell lung cancer & 10 & $\begin{array}{l}\text { CDK4, CDK2, LAMA3, COL4A1, } \\
\text { LAMB3, LAMC2, COL4A2, SKP2, }\end{array}$ & $3.81 \times 10^{-4}$ \\
\hline & KEGG & 00240 & Pyrimidine metabolism & 10 & $\begin{array}{l}\text { CCNE1, CDK6, NME1, UCK2, } \\
\text { TK1, POLA2, PRIM2, POLR3D, } \\
\text { POLE2, TYMP, TYMS, PRIM1 }\end{array}$ & $1.28 \times 10^{-3}$ \\
\hline & KEGG & 04115 & P53 signaling pathway & 8 & $\begin{array}{l}\text { SESN3, CDK4, CDK2, IGFBP3, } \\
\text { CHEK1, CCNB1, CCNE1, CDK6 }\end{array}$ & $1.61 \times 10^{-3}$ \\
\hline & KEGG & 04512 & $\begin{array}{l}\text { ECM-receptor } \\
\text { interaction }\end{array}$ & 8 & $\begin{array}{l}\text { SPP1, LAMA3, COL4A1, TNC, } \\
\text { LAMB3, LAMC2, COL4A2, ITGB4 }\end{array}$ & $5.98 \times 10^{-3}$ \\
\hline & KEGG & 05200 & Pathways in cancer & 18 & $\begin{array}{l}\text { CDK4, CDK2, LAMA3, PDGFB, } \\
\text { COL4A1, SLC2A1, LAMB3, } \\
\text { LAMC2, COL4A2, SKP2, BIRC5, } \\
\text { DVL3, EGFR, AR, WNT3, WNT7B, } \\
\text { CCNE1, CDK6 }\end{array}$ & $1.86 \times 10^{-2}$ \\
\hline & KEGG & 04510 & Focal adhesion & 11 & $\begin{array}{l}\text { SPP1, LAMA3, PDGFB, COL4A1, } \\
\text { TNC, LAMB3, LAMC2, COL4A2, } \\
\text { CAV2, EGFR, ITGB4 }\end{array}$ & $5.93 \times 10^{-3}$ \\
\hline Brown & KEGG & 04060 & $\begin{array}{l}\text { Cytokine-cytokine } \\
\text { receptor interaction }\end{array}$ & 7 & $\begin{array}{l}\text { CXCL12, LEPR, CCL15, CCL28, } \\
\text { CCL14, KIT, TNFRSF12A }\end{array}$ & $5.05 \times 10^{-2}$ \\
\hline Yellow & KEGG & 01100 & Metabolic pathways & 14 & $\begin{array}{l}\text { ATP6V0A4, FUT6, ST6GALNAC1, } \\
\text { GCNT3, ACSM3, EPHX2, AKR1B1, } \\
\text { GGT6, GALE, FUT2, MGLL, } \\
\text { TM7SF2, CYP3A5, B3GNT3 }\end{array}$ & $1.34 \times 10^{-2}$ \\
\hline
\end{tabular}

ECM, extracellular matrix; KEGG, Kyoto Encyclopedia of Genes and Genomes.

modules were merged, a total of 5 modules (appointed the black, brown, gray, pink and yellow modules to distinguish the 5 modules) were screened for the DEGs (Fig. 2). According to the result of the correlation analysis, their MS values were approximately the same (Table I). The pink and black modules contained mainly upregulated genes. By contrast, the brown 


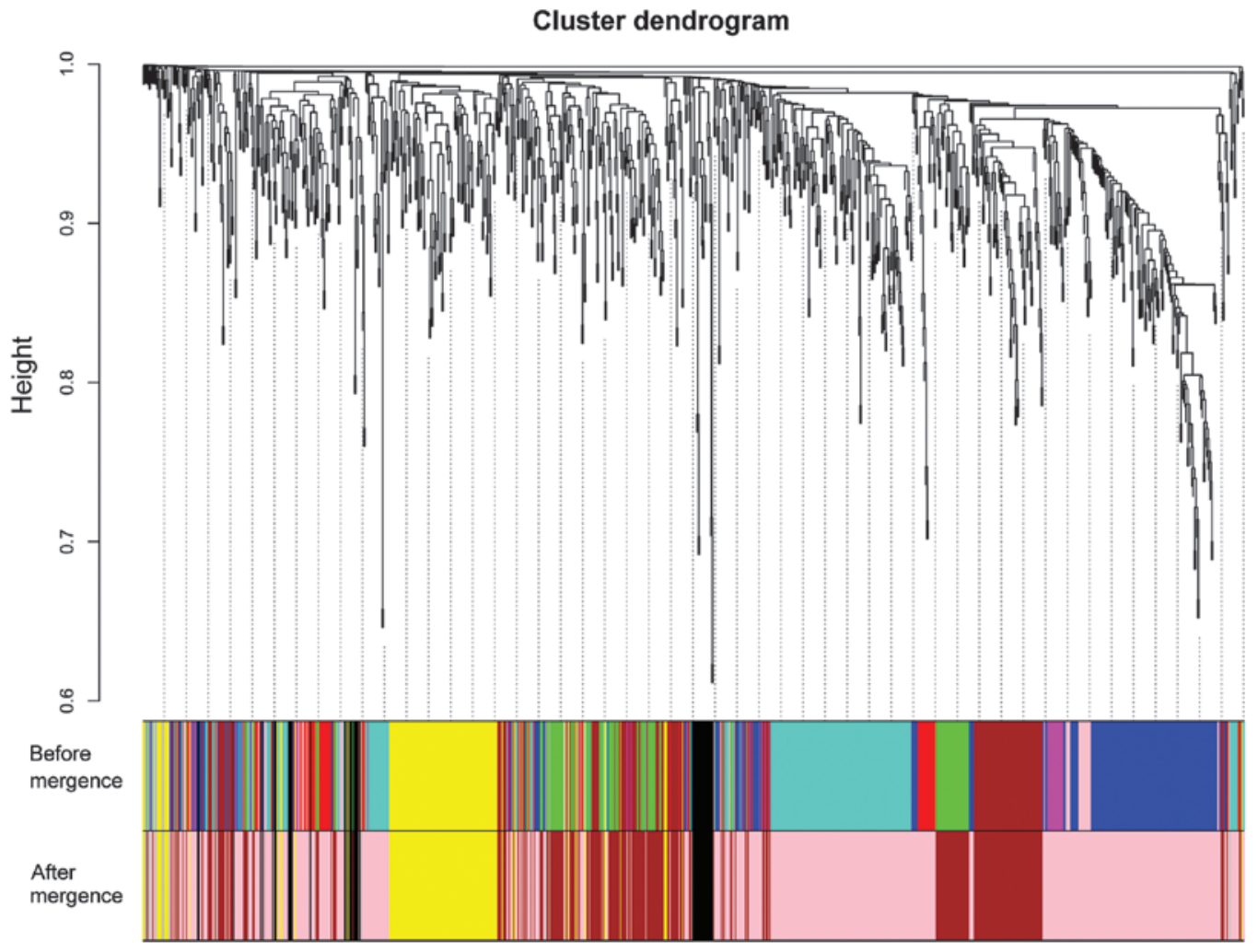

Figure 2. Clustering result prior to and after closed modules mergence.

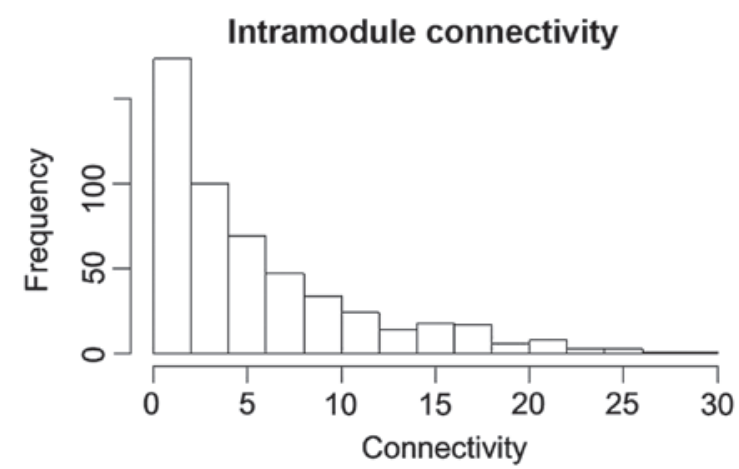

Figure 3. Connectivity distribution of differentially-expressed genes in the pink module.

and yellow modules mainly contained downregulated genes. Furthermore, the numbers of upregulated genes and downregulated genes were the same in the gray module.

Pathway enrichment analysis. Using the clusterProfiler package, pathway enrichment analyses were conducted separately for the DEGs in each module. However, only the DEGs in the brown, pink and yellow modules were involved in pathways. For the DEGs in the brown and yellow modules, the enriched pathways were the cytokine-cytokine receptor interaction and metabolic pathways, respectively. There were 13 enriched pathways for the DEGs in the pink module, including cell cycle $\left(\mathrm{P}=2.05 \times 10^{-8}\right)$, pathways in cancer $\left(\mathrm{P}=1.86 \times 10^{-2}\right)$ and focal adhesion $\left(\mathrm{P}=5.93 \times 10^{-2}\right)$ (Table II).

The connectivity distribution of the DEGs in the pink module is shown in Fig. 3. In the pink module, the DEGs with connectivity degrees $>20$ were upregulated in LSCC samples. In particular, TPX2, microtubule-associated homolog (TPX2; degree, 25), minichromosome maintenance complex component 2 (MCM2; degree, 25), ubiquitin-like with PHD and ring finger domains 1 (UHRF1; degree, 22), cyclin-dependent kinase $2(C D K 2$; degree, 20) and protein regulator of cytokinesis 1 (PRC1; degree, 20) exhibited high connectivity degrees in the pink module.

\section{Discussion}

In the present study, a total of 959 DEGs, including 553 upregulated genes and 406 downregulated genes, were screened from LSCC samples compared with adjacent non-cancerous samples. A total of 5 modules (the black, brown, gray, pink and yellow modules) were screened for the DEGs in the weighted co-expression network. The DEGs in the pink module were involved in the most pathways. TPX2 (degree, 25), MCM2 (degree, 25), UHRF1 (degree, 22), CDK2 (degree, 20) and PRC1 (degree, 20) may be of great importance in LSCC, as they had high connectivity degrees in the pink module.

As a serine-threonine kinase gene, Aurora-A may correlate with TPX2 during spindle assembly, and TPX2 functions in targeting Aurora-A to the spindle apparatus (20). Aurora-A and TPX2 are often co-overexpressed, therefore, certain functions of Aurora-A in cell transformation and tumorigenesis can be a result of the oncogenic activation of the Aurora-A/TPX2 complex (21). Upregulated Aurora-A may play important roles in the tumor progression and prognosis of head and neck squamous cell carcinoma (22). By enhancing the invasion ability and chromosomal instability, overexpressed Aurora-A may 
promote the carcinogenesis and progression of $\operatorname{LSCC}(4,23)$. Thus, the expression level of TPX2 may be associated with LSCC.

As a biomarker for showing the proliferation of laryngeal carcinoma cells, MCM2 can play a role in the occurrence, progression and prognosis of laryngeal carcinoma (24). It has been reported that overexpressed UHRF1 may function in the progression of LSCC and may be used as a promising marker for the prognosis of LSCC (25). By transforming cell cycle progression, promoting apoptosis and weakening the DNA damage repair capacity, the inhibition of UHRF1 can be implicated in the radioresistance of esophageal SCC (26). The results indicate that MCM2 and UHRF1 may have a close correlation with LSCC.

Cyclin D1, cyclin E and their catalytic subunits, $C D K 4$ and $C D K 2$, often are overexpressed in a number of human esophageal SCC cases (27). The overexpression of combined $C D K 2$ and proliferating cell nuclear antigen indicates a poor overall survival time, and $C D K 2$ expression may be associated with the biological behavior of LSCC (28). Overexpressed cyclin E has a correlation with poor clinicopathological parameters and can serve as an biomarker for cell proliferation and prognosis in patients with LSCC (29). These results may indicate that the expression level of $C D K 2$ is associated with LSCC. As a protein implicated in cytokinesis, $\mathrm{PRC} 1$ is a good in vivo substrate for several CDKs (30), indicating that PRCl may also play a role in LSCC through $C D K 2$.

In conclusion, the present study performed an integrated bioinformatics analysis of genes that may be associated with LSCC. A total of 959 DEGs were screened from LSCC samples compared with adjacent non-cancerous samples. Furthermore, TPX2, MCM2, UHRF1, CDK2 and PRC1 may play a role in LSCC. However, further studies are required to reveal their specific functions in LSCC.

\section{References}

1. Batsakis JG: Tumors of the head and neck: Clinical and pathological considerations. 2nd edition. Williams \& Wilkins, Baltimore, 1979.

2. Million R, Cassisi N and Clark J: Cancer of the head and neck. In: Cancer: Principles and Practice of Oncology. 3rd edition. JB Lippincott, PA, pp488-590, 1989.

3. Hunter KD, Parkinson EK and Harrison PR: Profiling early head and neck cancer. Nat Rev Cancer 5: 127-135, 2005.

4. Zhang H, Chen X, Jin Y, Liu B and Zhou L: Overexpression of Aurora-A promotes laryngeal cancer progression by enhancing invasive ability and chromosomal instability. Eur Arch Otorhinolaryngol 269: 607-614, 2012.

5. Bazan V, Zanna I, Migliavacca M, Sanz-Casla MT, Maestro ML, Corsale S, Macaluso M, Dardanoni G, Restivo S, Quintela PL, et al: Prognostic significance of p16INK4a alterations and 9p21 loss of heterozygosity in locally advanced laryngeal squamous cell carcinoma. J Cell Physiol 192: 286-293, 2002.

6. Rocco JW and Sidransky D: p16 (MTS-1/CDKN2/INK4a) in cancer progression. Exp Cell Res 264: 42-55, 2001.

7. Ogino T, Shigyo H, Ishii H, Katayama A, Miyokawa N, Harabuchi Y and Ferrone S: HLA class I antigen down-regulation in primary laryngeal squamous cell carcinoma lesions as a poor prognostic marker. Cancer Res 66: 9281-9289, 2006.

8. Dong Y, Sui L, Sugimoto K, Tai Y and Tokuda M: Cyclin D1-CDK4 complex, a possible critical factor for cell proliferation and prognosis in laryngeal squamous cell carcinomas. Int J Cancer 95: 209-215, 2001.

9. Vielba R, Bilbao J, Ispizua A, Zabalza I, Alfaro J, Rezola R, Moreno E, Elorriaga J, Alonso I, Baroja A and de la Hoz C: p53 and cyclin D1 as prognostic factors in squamous cell carcinoma of the larynx. Laryngoscope 113: 167-172, 2003.
10. Lauriola L, Michetti F, Maggiano N, Galli J, Cadoni G, Schäfer BW, Heizmann CW and Ranelletti FO: Prognostic significance of the $\mathrm{Ca}(2+)$ binding protein $\mathrm{S} 100 \mathrm{~A} 2$ in laryngeal squamous-cell carcinoma. Int J Cancer 89: 345-349, 2000.

11. Sun Y, Liu M, Yang B, Li B and Lu J: Role of siRNA silencing of MMP-2 gene on invasion and growth of laryngeal squamous cell carcinoma. Eur Arch Otorhinolaryngol 265: 1385-1391, 2008.

12. Liu WW, Zeng ZY, Wu QL, Hou JH and Chen YY: Overexpression of MMP-2 in laryngeal squamous cell carcinoma: A potential indicator for poor prognosis. Otolaryngol Head Neck Surg 132: 395-400, 2005

13. Cao WF, Zhang LY, Liu MB, Tang PZ, Liu ZH and Sun BC: Prognostic significance of stomatin-like protein 2 overexpression in laryngeal squamous cell carcinoma: Clinical, histologic and immunohistochemistry analyses with tissue microarray. Hum Pathol 38: 747-752, 2007.

14. Cao WF, Zhang LY, Zhang B, Liu MB, Liu ZH and Sun BC: Relationship between SLP-2 expression and prognosis in laryngeal squamous cell carcinoma and mammary invasive carcinoma. Zhonghua Bing Li Xue Za Zhi 39: 332-337, 2010 (In Chinese).

15. Smyth GK: Limma: Linear models for microarray data. In: Bioinformatics and Computational Biology Solutions using $\mathrm{R}$ and Bioconductor. Gentleman R, Carey V, Dudoit S, Irizarry R and Huber W (eds.), Springer, New York, pp397-420, 2005.

16. Langfelder $P$ and Horvath S: WGCNA: An R package for weighted correlation network analysis. BMC Bioinformatics 9: $559,2008$.

17. Janssens F, Zhang L, De Moor B and Glänzel W: Hybrid clustering for validation and improvement of subject-classification schemes. Inform Process Manag 45: 683-702, 2009

18. Kanehisa M, Goto S, Sato Y, Furumichi M and Tanabe M: KEGG for integration and interpretation of large-scale molecular data sets. Nucleic Acids Res 40 (Database Issue): D109-D114, 2012.

19. Yu G, Wang LG, Han Y and He QY: ClusterProfiler: An $\mathrm{R}$ package for comparing biological themes among gene clusters. OMICS 16: 284-287, 2012.

20. Kufer TA, Silljé HH, Körner R, Gruss OJ, Meraldi P and Nigg EA: Human TPX2 is required for targeting Aurora-A kinase to the spindle. J Cell Biol 158: 617-623, 2002.

21. Asteriti IA, Rensen WM, Lindon C, Lavia P and Guarguaglini G: The Aurora-A/TPX2 complex: A novel oncogenic holoenzyme? Biochim Biophys Acta 1806: 230-239, 2010.

22. Reiter R, Gais P, Jütting U, Steuer-Vogt MK, Pickhard A, Bink K, Rauser S, Lassmann S, Höfler H, Werner $M$ and Walch A: Aurora kinase A messenger RNA overexpression is correlated with tumor progression and shortened survival in head and neck squamous cell carcinoma. Clin Cancer Res 12: 5136-5141, 2006.

23. Guan Z, Wang XR, Zhu XF, Huang XF, Xu J, Wang LH, Wan XB, Long ZJ, Liu JN, Feng GK, et al: Aurora-A, a negative prognostic marker, increases migration and decreases radiosensitivity in cancer cells. Cancer Res 67: 10436-10444, 2007.

24. Chatrath P, Scott IS, Morris LS, Davies RJ, Rushbrook SM, Bird K, Vowler SL, Grant JW, Saeed IT, Howard D, et al: Aberrant expression of minichromosome maintenance protein-2 and Ki67 in laryngeal squamous epithelial lesions. Br J Cancer 89: 1048-1054, 2003.

25. Pi JT, Lin Y, Quan Q, Chen LL, Jiang LZ, Chi W and Chen HY: Overexpression of UHRF1 is significantly associated with poor prognosis in laryngeal squamous cell carcinoma. Med Oncol 30: 613,2013 .

26. Yang C, Wang Y, Zhang F, Sun G, Li C, Jing S, Liu Q and Cheng Y: Inhibiting UHRF1 expression enhances radiosensitivity in human esophageal squamous cell carcinoma. Mol Biol Rep 40: 5225-5235, 2013.

27. Matsumoto M, Furihata M, Ishikawa T, Ohtsuki Y and Ogoshi S: Comparison of deregulated expression of cyclin D1 and cyclin $\mathrm{E}$ with that of cyclin-dependent kinase 4 (CDK4) and CDK2 in human oesophageal squamous cell carcinoma. Br J Cancer 80: 256-261, 1999

28. Dong Y, Sui L, Tai Y, Sugimoto K and Tokuda M: The overexpression of cyclin-dependent kinase (CDK) 2 in laryngeal squamous cell carcinomas. Anticancer Res 21: 103-108, 2000.

29. Dong Y, Sui L, Tai Y, Sugimoto K, Hirao T and Tokuda M: Prognostic significance of cyclin $\mathrm{E}$ overexpression in laryngeal squamous cell carcinomas. Clin Cancer Res 6: 4253-4258, 2000.

30. Jiang W, Jimenez G, Wells NJ, Hope TJ, Wahl GM, Hunter T and Fukunaga R: PRC1: A human mitotic spindle-associated CDK substrate protein required for cytokinesis. Mol Cell 2: 877-885, 1998. 